University of Nebraska - Lincoln

DigitalCommons@University of Nebraska - Lincoln

6-1-1981

\title{
A unidirectional, pulsed far-infrared ring laser
}

\author{
W.A. Peebles \\ University of California, Los Angeles \\ Donald P. Umstadter \\ University of Nebraska-Lincoln, donald.umstadter@unl.edu \\ D.L. Brower \\ University of California, Los Angeles \\ N.C. Luhmann Jr. \\ University of California, Los Angeles
}

Follow this and additional works at: https://digitalcommons.unl.edu/physicsumstadter

Part of the Physics Commons

Peebles, W.A.; Umstadter, Donald P.; Brower, D.L.; and Luhmann, N.C. Jr., "A unidirectional, pulsed farinfrared ring laser" (1981). Donald Umstadter Publications. 39.

https://digitalcommons.unl.edu/physicsumstadter/39

This Article is brought to you for free and open access by the Research Papers in Physics and Astronomy at DigitalCommons@University of Nebraska - Lincoln. It has been accepted for inclusion in Donald Umstadter Publications by an authorized administrator of DigitalCommons@University of Nebraska - Lincoln. 


\title{
A unidirectional, pulsed far-infrared ring laser
}

\author{
W. A. Peebles, D. Umstadter, D. L. Brower, and N. C. Luhmann, Jr. \\ University of California, Los Angeles, California 90024
}

(Received 23 February 1981; accepted for publication 23 March 1981)

The first study of a pulsed, far-infrared, ring laser is described. Unidirectional, traveling-wave operation is observed, thereby eliminating the spatial hole burning effects present in linear cavities. Single-mode output powers of $100 \mathrm{~kW}$ have been obtained.

PACS numbers: 42.60.By, 42.60.Da, 52.70.Kz

Standing-wave electric field patterns existing in linear laser cavities result in a spatially inhomogeneous population inversion. This encourages the simultaneous oscillation of several longitudinal cavity modes even when the lasing transition is homogeneously broadened. Unidirectional, traveling-wave ring lasers eliminate these spatial inhomogeneity effects and thus offer possible improvements in mode quality and efficiency. Optically pumped, far-infrared (FIR) lasers possess an additional advantage from the use of a ring cavity, since direct feedback of the pump radiation existing in linear systems is automatically eliminated. This characteristic has already been utilized in cw FIR systems, where significant improvements in amplitude stability have been achieved. ${ }^{1}$

The development of a high-power $(\simeq 1 \mathrm{MW})$ narrow bandwidth ( $\leqslant 50 \mathrm{MHz}$ ), pulsed FIR laser for diagnostic research of fusion plasmas has been an area of great activity over the last few years. ${ }^{2.3}$ However, all of the schemes investigated have consisted of either stable or unstable linear cavities ${ }^{4-8}$ This letter describes the first study of a high-power, pulsed FIR ring laser. In this instance the lasing transition is homogeneously broadened by collisions and so differs from the low pressure, Doppler-broadened cw systems. The prime motivation is improvement in mode quality and not solely the elimination of pump feedback effects.

In order to investigate the pulsed ring laser configuration and also compare performance with a folded linear system, the experimental arrangement illustrated schematically in Fig. 1 was adopted. A grating-tuned Lumonics 602 TEA, $\mathrm{CO}_{2}$ laser producing up to $\simeq 40 \mathrm{~J}$ on the $\mathrm{R} 22(\lambda=9.26 \mu \mathrm{m})$ line comprised the pump source. The laser was operated with high $\mathrm{N}_{2}$ levels producing a pump pulse characterized by an initial high-power spike ( $\tau \simeq 80 \mathrm{~ns}, P \simeq 150 \mathrm{MW})$ followed by a lower level $1.5-\mu$ s tail. This ensured that the FIR medium was pumped for a long period compared to the roundtrip propagation times of the cavities investigated. The infrared output beam was first reduced to $\simeq 50 \mathrm{~mm}$ diameter

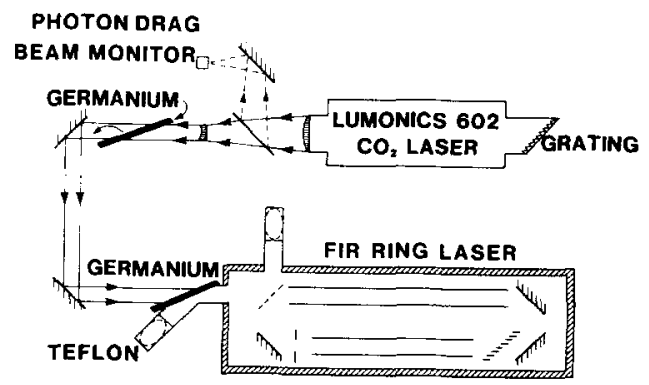

FIG. 1. Experimental arrangement. and was then incident on a germanium beamsplitter, which upon rotation allowed easy and continuous variation of the incident power. The transmitted $\mathrm{CO}_{2}$ radiation entered the FIR laser through a Germanium vacuum window tilted at Brewster's angle.

The FIR system consisted of a $160 \times 65 \times 25-\mathrm{cm}$ evacuated aluminum box, which could be filled to any desired operating pressure with an absorbing molecular gas. In this letter results are presented for the $385 / 359-\mu \mathrm{m}$ emission from $\mathrm{D}_{2} \mathrm{O} .{ }^{8}$ Internal to the box a variety of cavity configurations could be easily studied thereby making comparison a relatively simple and meaningful procedure. The FIR ring cavity consisted of three flat copper mirrors together with an electroformed mesh $(60$ lines $/ \mathrm{cm})$ output coupler. The total cavity length was $\simeq 2 \mathrm{~m}$, although variation up to $\simeq 5 \mathrm{~m}$ was possible. Dielectric waveguide ( $50 \mathrm{~mm}$ diameter, $60 \mathrm{~cm}$ long) was used in the long arms of the ring to aid FIR mode control and also confinement of the $\mathrm{CO}_{2}$ pump radiation. Conversion to a folded linear system was easily achieved by a $45^{\circ}$ rotation of the output coupler and final copper mirror. In addition, superfluorescent operation could easily be studied by complete removal of the mesh output coupler.

FIR radiation polarized perpendicular to the $\mathrm{CO}_{2}$ pump and traveling in the backward direction (relative to the $\mathrm{CO}_{2}$ pump) is reflected ( $\simeq 80 \%$ ) by the input germanium window and coupled out through a Brewster-angled Teflon window. In the forward direction, both parallel and perpendicular polarized radiation are coupled out directly. In the above manner, feedback of FIR radiation to the ring oscillator is minimized, thereby preventing the development of spurious standing-wave oscillations.

Energy measurements were made using a model 3620 Scientech colorimeter. Foote and Hodges ${ }^{9}$ have determined a calibration factor of 1.9 at $447 \mu \mathrm{m}$, and this factor has therefore been adopted throughout this work. The time history of the FIR emission was recorded using a quasioptical, biconical, GaAs Schottky-barrier diode detector. ${ }^{10}$ The bandwidth of the detection system was set by the Tektronix 7844 oscilloscope ( $400 \mathrm{MHz}$ ).

Initially superfluorescent emission was studied. Peak output energies of $16 \mathrm{~mJ}$ in the forward and $9.5 \mathrm{~mJ}$ in the backward direction were measured. In addition, the ratio of perpendicular to parallel $(\perp / \|)$ polarized emission in the forward direction was $\simeq 2$. Since only $\perp$ polarized emission is observed in the backward direction, this implies that the FIR emission is virtually equal in each direction. The time history of the emission indicated random, high-frequency modulation typical of superfluorescence. 
Conversion to a ring oscillator using a $60-$ line $/ \mathrm{cm}$ electroformed inductive mesh resulted in improved efficiency. An output energy of $\simeq 90 \mathrm{~mJ}$ was measured in the forward direction as compared to less than $0.8 \mathrm{~mJ}$ in the backward direction. The relative increase in forward emission is a result of "seeding" the oscillator in the forward direction via the small amount of superfluorescent emission generated external to the cavity. This effect has ensured unidirectional, traveling-wave operation of the pulsed ring laser and therefore eliminates the possibility of spatial hole burning effects.

Similar to the superfluorescent case, the ratio of $1 / \|$ polarized emission in the forward direction was $\simeq 2$ for the ring oscillator. Simultaneous measurement of the time history of both polarization components was made using a polarizer and two Schottky diode detectors. Initially, these measurements suggested significant random structure.

However, closer investigation revealed bursts of coherent oscillations ranging in frequency from $\simeq 2$ to $50 \mathrm{MHz}$. The longitudinal mode frequency for the cavity was $\simeq 150 \mathrm{MHz}$, and so it was assumed that the observed oscillations were the result of transverse-mode beating. It should also be noted that the two polarization signals were not correlated on either short or long time scales. Peak-to-tail ratios of the output power were $\simeq 1$ for perpendicularly polarized emission and $\simeq 2$ for parallel. A similar trend was also observed for superfluorescent emission, indicating that the effect was probably a result of gain saturation.

To eliminate any possible confusion due to the simultaneous existence of two polarizations, a grid polarizer $(140 / \mathrm{cm})$ was added internal to the cavity as indicated in Fig. 1. The grid was oriented to pass only $\perp$ polarization. The FIR output energy was reduced to $\sim 60 \mathrm{~mJ}$ in the forward direction and remained $<0.8 \mathrm{~mJ}$ in the backward direction.

In order to increase diffraction losses for the higherorder transverse modes, a 30-mm aperture was placed on axis in the FIR cavity. A comparison of the FIR emission, with and without the aperture is shown in Fig. 2 at a $\mathrm{D}_{2} \mathrm{O}$ pressure of 7 Torr. Inclusion of the aperture in the ring cavity has eliminated transverse mode beating and resulted in a temporally smooth output characteristic of single-mode operation. Single-mode operation was found to persist as the pressure was varied from 12 to 1 Torr with a maximum output energy of $20 \mathrm{~mJ}(100 \mathrm{~kW})$ being obtained at $\sim 7$ Torr. However, at fill pressures of $\leqslant 0.5$ Torr a strong modulation characteristic of longitudinal mode beating (150 MHz) was observed as illustrated in Fig. 3. At high pressures the FIR
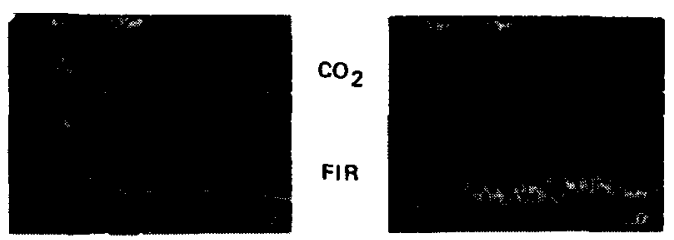

$100 \mathrm{~ns} / \mathrm{div}$

$30 \mathrm{~mm}$ APERTURE

NO APERTURE

FIG. 2. Time history of the $2-\mathrm{m}$-long ring oscillator emission with and without an internal aperture. The limiting bandwidth was set by the oscilloscope at $400 \mathrm{MHz}$.
12.5 TORR

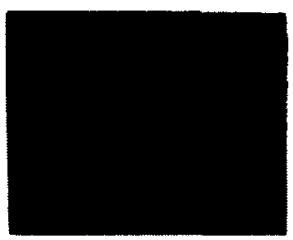

$200 \mathrm{~ns} / \mathrm{div}$.
3 TORR

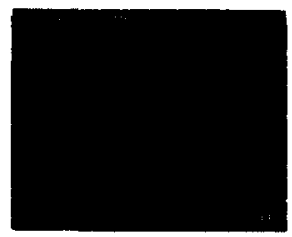

$200 \mathrm{~ns} /$ div.
0.5 TORR

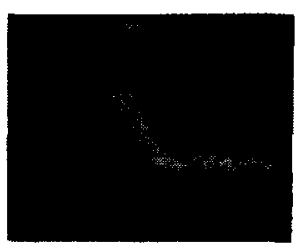

$50 \mathrm{~ns} / \mathrm{div}$.

FIG. 3. Time history of ring oscillator output as a function of fill pressure. Longitudinal mode beating is observed at pressures $\leqslant 0.5$ Torr.

lasing medium is homogeneously broadened. As the pressure is lowered, inhomogeneous broadening mechanisms, such as Doppler broadening, become significant and therefore the possibility of multilongitudinal mode operation is increased.

Conversion of the ring oscillator into a 2-m folded linear system resulted in maximum output energies of $\simeq 100$ $\mathrm{mJ}$. The FIR emission was reduced to $\sim 25 \mathrm{~mJ}$ with the inclusion of the polarizing grid and $30-\mathrm{mm}$ aperture. These output energies are similar to that obtained from the ring cavity, suggesting similar energy conversion efficiencies. This can be contrasted with cw FIR systems where linear cavities appear to be twice as efficient. ${ }^{11}$

A comparison of FIR pulse shapes from the ring and linear systems, both with a $30-\mathrm{mm}$ aperture and polarizing grid, is shown in Fig. 4 at a fill pressure of 7 Torr. The linear laser continues to exhibit multimode output, while the ring laser emission is single mode. Further reduction of the aperture diameter to $15 \mathrm{~mm}$ failed to eliminate the modulation, whose frequency $(70 \mathrm{MHz})$ was characteristic of longitudinal mode beating. In addition, reduction of the cavity length to produce the same round-trip time as the ring cavity failed to significantly improve the mode quality of the linear laser.

In conclusion, the first study of a pulsed FIR ring laser has demonstrated that unidirectional, traveling-wave operation can be achieved via external seeding of the cavity. The

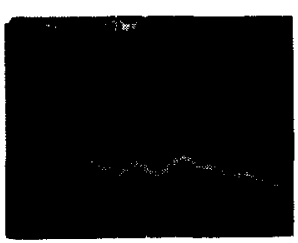

RING

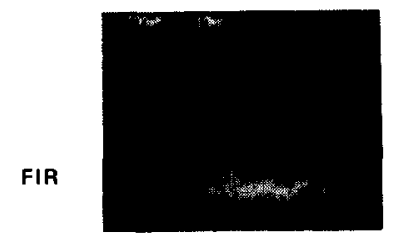

LINEAR
100 ns/div.

FIG. 4. Comparison of temporal output from ring and linear cavities. The modulation frequency on the linear emission corresponds to longitudinal mode beating for the linear cavity. (Grid polarizer and $30-\mathrm{mm}$ aperature internatul to both oscillators. 
spatial hole burning effects present in linear cavities are therefore eliminated. A comparison between ring and folded linear systems has demonstrated the mode quality of the ring to be significantly superior. Single-mode output powers of $\simeq 100 \mathrm{~kW}$ have been obtained with efficiencies comparable to the multimode output from the folded linear cavity.

We wish to thank A. Semet for providing the grid polarizer used in this work and $J$. Hardy for his expert contacting of the Schottky mixers. Useful discussions with L. Casperson are also gratefully acknowledged. This work was supported by the U. S. Department of Energy, Office of Fusion Energy under Contract No. DE-AM03-76-SF00010.

'J. Heppner and C. O. Weiss, Appl. Phys. Lett. 33, 590 (1978).

${ }^{2} \mathrm{~N}$. C. Luhmann, Jr. , Infrared and Millimeter Waves, Vol. 1, edited by K.
J. Button (Academic, New York, 1979), p. 1-65.

${ }^{3}$ D. L. Jassby, D. R. Cohn, B. Lax, and W. Halverson, Nucl. Fusion 14, 745 (1974).

${ }^{4}$ D. T. Hodges, Infrared Phys. 18, 375 (1978).

${ }^{5}$ T. A. De Temple, SPIE Seminar on Far Infrared/ Submillimeter Tech. and Appl., SPIE 105, 11 (1977)

${ }^{6} \mathrm{~L}$. C. Johnson and A. Semet, Fifth International Conference on Infrared and Millimeter Waves, Paper W9.5, Wurzburg, 1980 (unpublished).

D. E. Evans, L. E. Sharp, W. A. Peebles and G. Taylor, IEEE J. Quantum Electron. QE-13, 54 (1977).

${ }^{8}$ P. Woskoboinikow, H. C. Praddaude, W. J. Mulligan, D. R. Cohn, and B. Lax, J. Appl. Phys. 50, 1125 (1979).

${ }^{9}$ F. B. Foote and D. T. Hodges, Fourth International Conference on Infrared and Millimeter Waves, Florida, IEEE Cat. No. 1384-7 MTT, 78, 1979 (IEEE, New York, 1979).

'"J. J. Gustincic, 1977 IEEE MITT-S International Microwave Symposium Digest, IEEE Cat. No. CH1219-5 MITT, 99, 1977 (IEEE, New York, 1977).

''Y. C. Ni, Appl. Opt. 19, 3425 (1980). 